\title{
Numerical Study of a Microscopic Artificial Swimmer
}

\author{
Erik Gauger $^{1}$ and Holger Stark ${ }^{1,2}$ \\ ${ }^{1}$ Fachbereich Physik, Universität Konstanz, D-78457 Konstanz, Germany \\ ${ }^{2}$ Max-Planck-Institut für Dynamik und Selbstorganisation, Bunsenstr. 10, D-37073 Göttingen, Germany
}

(Dated: October 24, 2018)

\begin{abstract}
We present a detailed numerical study of a microscopic artificial swimmer realized recently by Dreyfus et al. in experiments [R. Dreyfus et al., Nature 437, 862 (2005)]. It consists of an elastic filament composed of superparamagnetic particles that are linked together by DNA strands. Attached to a load particle, the resulting swimmer is actuated by an oscillating external magnetic field so that it performs a non-reciprocal motion in order to move forward. We model the superparamagnetic filament by a bead-spring configuration that resists bending like a rigid rod and whose beads experience friction with the surrounding fluid and hydrodynamic interactions with each other. We show that, aside from finite-size effects, its dynamics is governed by the dimensionless sperm number, the magnitude of the magnetic field, and the angular amplitude of the field's oscillating direction. Then we study the mean velocity and the efficiency of the swimmer as a function of these parameters and the size of the load particle. In particular, we clarify that the real velocity of the swimmer is influenced by two main factors, namely the shape of the beating filament (determined by the sperm number and the magnetic-field strength) and the oscillation frequency. Furthermore, the load size influences the performance of the swimmer and has to be chosen as a compromise between the largest swimming velocity and the best efficiency. Finally, we demonstrate that the direction of the swimming velocity changes in a symmetry-breaking transition when the angular amplitude of the field's oscillating direction is increased, in agreement with experiments.
\end{abstract}

PACS numbers: 87.19.St, 87.16.Ac,87.16.Qp

\section{INTRODUCTION}

Nature was very inventive to design mechanisms that microorganisms such as bacteria and many eukaryotic cells use to propel themselves in a highly viscous environment, i. e., at very low Reynolds numbers [1]. Since they cannot rely on drifting by inertia, as we do when we swim in water, they immediately come to a halt when they stop with their beating motion. In this article we study in detail a microscopic artificial swimmer [2] that was constructed recently on the basis of a superparamagnetic elastic filament that mimics the so-called flagellum employed by many eukaryotic cells [1].

In 1977 Purcell pointed out in his famous article "Life at low Reynolds number" that microorganisms have to perform a non-reciprocal periodic motion to be able to move forward [3] (see also [4]). Non-reciprocal means that the time-reversed motion is not the same as the original one (for examples see [5, 6, 7, 8, 9, 10]). The reason lies in the Stokes equations [11] governing the fluid flow around the microorganisms for negligible inertia: they allow for a time-inverted flow pattern when all the external forces are inverted.

Bacteria employ a marvelous rotary motor to crank a relatively stiff helical filament [12, 13]. Spermatozoa as one example for eukaryotic cells move forward by creating bending waves that move along their elastic flagella from the head to the tail [14, 15, 16]. While these waves are generated by a collective motion of internal molecular motors [17, 18], Dreyfus et al. use an external magnetic field to induce the beating of a superparamagnetic filament attached to a red-blood cell [2]. The filaments are made from superparamagnetic colloidal particles of micron size. A static external magnetic field induces dipoles in the colloids so that they form a chain. In the gaps between the charged colloids chemical linkers such as doublestranded DNA are attached to the particles and an elastic filament resisting bending and stretching is formed [19, 20, 21] (for similar systems see [22]). As demonstrated by the impressive experiments of Dreyfus et al., an oscillating external magnetic field now induces a non-reciprocal beating motion of the superparamagnetic filament that is able to move the attached red-blood cell forward.

So far, the modelling of the dynamics of the superparamagnetic filament followed the elastohydrodynamics of an elastic rod [18, 23] supplemented by a continuum version for the interaction of the magnetic-field induced dipoles [2, 24] or a simpler description for the interaction with the magnetic field [25]. The authors are able to describe the dynamics of the filament [24, 25] and the velocity curve of the artificial swimmer [2]. Here we present a different description of the artificial swimmer similar to the one used by Lagomarsino and Lowe for driven microfilaments [26, 27]. We take into account the discrete nature of the superparamagnetic filament by modelling it as a sequence of beads, and, in contrast to the work of Roper et al. [24], we consider dipolar and hydrodynamic interactions between all the beads. We present a thorough investigation of the swimming velocity and efficiency as a function of the relevant parameters, study the influence of the size of the load attached to the filament, and demonstrate that the swimmer can take different directions via a symmetrybreaking transition depending on how the actuating magnetic field oscillates.

In Sec. [I] we present details for the modeling of the dynamics of the superparamagnetic filament and show that aside from finite-size effects it is governed by a few relevant parameters. Section $\amalg$ summarizes and discusses the results from our numerical study and Sec. IV contains our concluding remarks. 


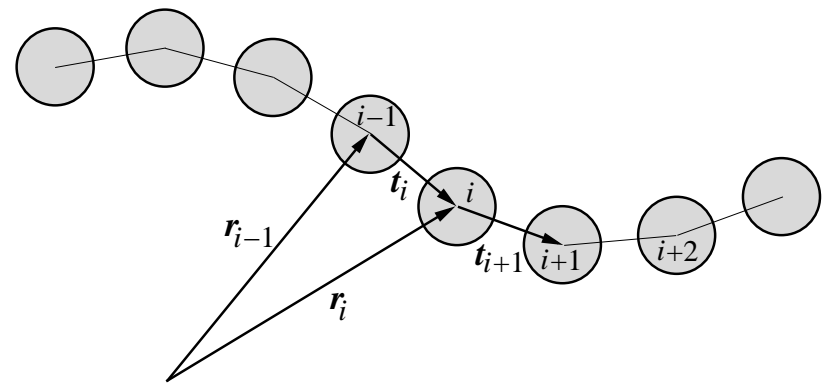

FIG. 1: Bead-spring model for the superparamagnetic filament consisting of $N$ particles. Each bead at position $\boldsymbol{r}_{i}$ experiences forces due to bending and stretching and due to interactions of the magneticfield induced dipoles located on the beads.

\section{MODELLING THE SUPERPARAMAGNETIC FILAMENT}

We model the superparamagnetic filament by a bead-spring configuration, as illustrated in Fig. 1 that, in addition, resists bending like a worm-like chain [28]. Thus each bead in the filament experiences a force due to stretching, bending and dipolar interactions. While the chemical linkers between the beads are responsible for the resistance to bending and stretching, we completely ignore their contribution to hydrodynamic friction. So the filament interacts with the fluid surrounding via the hydrodynamic friction of the beads that incorporates their hydrodynamic interactions. In the following we first set up the discretized version of the bending and stretching free energies [29] and also formulate the dipolar interaction energy from which we then calculate the forces on a single bead. In a second step, the equations of motion for the single beads are established with the help of their mobilities on the RotnePrager level. Comments on numerical details and the scaling behavior conclude this section.

\section{A. Energies and Forces}

A deviation of the distance $l_{i}=\left|\boldsymbol{t}_{i}\right|$ of the beads from their equilibrium value $l_{0}$ obeys Hooke's law and the total stretching free energy is

$$
H^{S}=\frac{1}{2} k \sum_{i=2}^{N}\left(l_{i}-l_{0}\right)^{2},
$$

where $N$ denotess the total number of beads. Typically, we consider relatively stiff springs so the variation of $l_{i}$ is always smaller than $0.1 l_{0}$. The stretching force acting on bead $i$ obeys

$$
\boldsymbol{F}_{i}^{S}=-\nabla_{\boldsymbol{r}_{i}} H^{S}=-k\left(l_{i}-l_{0}\right) \hat{\boldsymbol{t}}_{i}+k\left(l_{i+1}-l_{0}\right) \hat{\boldsymbol{t}}_{i+1},
$$

where $\nabla_{\boldsymbol{r}_{i}}$ is the nabla operator with respect to $\boldsymbol{r}_{i}$.

The linkers connecting the beads convey some bending rigidity to the filament. The bending free energy of an elastic rod or a worm-like chain is given by [30]

$$
H^{B}=\frac{1}{2} A \int_{0}^{L} d s\left(\frac{d \hat{\boldsymbol{t}}}{d s}\right)^{2}
$$

where $L$ is the total length of the filament, $s$ the arclength along it, and $\hat{t}$ the unit tangent at location $s$. Refering the bending stiffness $A=k_{\mathrm{B}} T l_{p}$ to the thermal energy $k_{\mathrm{B}} T$, one obtains the persistence length $l_{p}$ that gives the length scale on which the filament becomes flexible. Replacing $d \hat{\boldsymbol{t}} /(d s)$ by $\left(\hat{t}_{i+1}-\hat{\boldsymbol{t}}_{i}\right) / l_{0}$, we arrive at the discretized version of the bending energy for the superparamagnetic filament,

$$
H^{B}=\frac{A}{l_{0}} \sum_{i=1}^{N} f_{i}\left(1-\hat{\boldsymbol{t}}_{i+1} \cdot \hat{\boldsymbol{t}}_{i}\right),
$$

where $\hat{\boldsymbol{t}}_{i}=\boldsymbol{t}_{i} / l_{i}$. We have introduced the factor

$$
f_{i}=\left\{\begin{array}{l}
1 \text { for } 2 \leq i \leq N-1 \\
0 \text { for } i=1, N
\end{array}\right.
$$

to let the index $i$ in Eq. (4) still run from 1 to $N$, i. e., over all beads. This facilitates the calculation of the bending force $\boldsymbol{F}_{i}^{B}=-\nabla_{\boldsymbol{r}_{i}} H^{B}$ acting on each bead $i$ :

$$
\begin{aligned}
\boldsymbol{F}_{i}^{B} & =\frac{A}{l_{0}}\left\{\frac{f_{i-1}}{l_{i}} \hat{\boldsymbol{t}}_{i-1}-\left[\frac{f_{i-1}}{l_{i}} \hat{\boldsymbol{t}}_{i-1} \cdot \hat{\boldsymbol{t}}_{i}+\frac{f_{i}}{l_{i+1}}+\frac{f_{i}}{l_{i}} \hat{\boldsymbol{t}}_{i} \cdot \hat{\boldsymbol{t}}_{i+1}\right] \hat{\boldsymbol{t}}_{i}\right. \\
& \left.+\left[\frac{f_{i}}{l_{i+1}} \hat{\boldsymbol{t}}_{i} \cdot \hat{\boldsymbol{t}}_{i+1}+\frac{f_{i}}{l_{i}}+\frac{f_{i+1}}{l_{i+1}} \hat{\boldsymbol{t}}_{i+1} \cdot \hat{\boldsymbol{t}}_{i+2}\right] \hat{\boldsymbol{t}}_{i+1}-\frac{f_{i+1}}{l_{i+1}} \hat{\boldsymbol{t}}_{i+2}\right\}
\end{aligned}
$$

To arrive at $\boldsymbol{F}_{i}^{B}$ we have used the relations $\boldsymbol{\nabla}_{\boldsymbol{r}_{i}} \hat{\boldsymbol{t}}_{i}=\frac{1}{l_{i}}\left(\mathbf{1}-\hat{\boldsymbol{t}}_{i} \otimes\right.$ $\left.\hat{\boldsymbol{t}}_{i}\right)$ and $\boldsymbol{\nabla}_{\boldsymbol{r}_{i}} \hat{\boldsymbol{t}}_{i+1}=-\frac{1}{l_{i+1}}\left(\mathbf{1}-\hat{\boldsymbol{t}}_{i+1} \otimes \hat{\boldsymbol{t}}_{i+1}\right)$ where $\mathbf{1}$ is the unit tensor and the symbol $\otimes$ means tensor product.

A single particle made from material with magnetic susceptibility $\chi$ and subjected to an external magnetic field $\boldsymbol{B}$ (more correctly the magnetic induction) aquires a magnetic dipole moment

$$
p=\frac{4 \pi a^{3}}{3 \mu_{0}} \chi B
$$

where $\mu_{0}=4 \pi \cdot 10^{-7} \mathrm{~N} / \mathrm{A}^{2}$ is the permeability of free space and $a$ is the particle's radius. In the filament, the dipole moments $\boldsymbol{p}_{i}$ of the beads interact with each other with a total dipole-dipole interaction energy

$$
H^{D}=\frac{\mu_{0}}{4 \pi} \sum_{i, j=1}^{N} \frac{\boldsymbol{p}_{i} \cdot \boldsymbol{p}_{j}-3\left(\boldsymbol{p}_{i} \cdot \hat{\boldsymbol{r}}_{i j}\right)\left(\boldsymbol{p}_{j} \cdot \hat{\boldsymbol{r}}_{i j}\right)}{r_{i j}^{3}} .
$$

where $r_{i j}=\left|\boldsymbol{r}_{j}-\boldsymbol{r}_{i}\right|, \hat{\boldsymbol{r}}_{i j}=\left(\boldsymbol{r}_{j}-\boldsymbol{r}_{i}\right) / r_{i j}$ and $\Sigma^{\prime}$ means sum over all $i \neq j$. When all the beads experience the same magnetic field $\boldsymbol{B}=\boldsymbol{B} \hat{\boldsymbol{p}}$, the total interaction energy becomes

$$
H^{D}=\frac{4 \pi a^{6}}{9 \mu_{0}}(\chi B)^{2} \sum_{i, j=1}^{N}, \frac{1-3\left(\hat{\boldsymbol{p}} \cdot \hat{\boldsymbol{r}}_{i j}\right)}{r_{i j}^{3}} .
$$

Here we ignore that the local magnetic field differs from the external $\boldsymbol{B}$ since all the induced dipoles contribute to the local field. Taking into account the field from the nearest neighbors of a dipole, one can show that this renormalizes the susceptibility $\chi$ and gives it a small anisotropy with $\chi_{\|}-\chi_{\perp} \approx-0.25$ for a typical value of $\chi \approx 1$, where $\|$ and $\perp$ refer, respectively, to directions parallel and perpendicular to the local filament 
axis [24]. In the following we are interested in the basic features of the system and therefore work with the energy (9) to calculate the force $\boldsymbol{F}_{i}^{D}=-\nabla_{r_{i}} H^{D}$ that all magnetic dipoles excert on bead $i$ :

$$
\boldsymbol{F}_{i}^{D}=\frac{4 \pi a^{6}}{3 \mu_{0}}(\chi B)^{2} \sum_{j=1}^{N} \frac{2\left(\hat{\boldsymbol{p}} \cdot \hat{\boldsymbol{r}}_{i j}\right) \hat{\boldsymbol{p}}+\left[1-5\left(\hat{\boldsymbol{p}} \cdot \hat{\boldsymbol{r}}_{i j}\right)^{2}\right] \hat{\boldsymbol{r}}_{i j}}{r_{i j}^{4}} .
$$

The filament is now driven by an external field whose direction $\hat{\boldsymbol{p}}(t)$ and magnitude $B(t)$ depend on time. For example, if the direction is oscillating very slowly, the filament will follow the field like a rigid rod since the induced dipoles prefer to align themselves along a common line. However, if the oscillations become faster, the hydrodynamic friction of the beads with the surrounding fluid increases so that they are not fast enough to form a straight line and the filament is bending.

\section{B. Equations of Motion}

The filament is immersed in a viscous fluid such as water. On the length scale of microns and for times larger than the momentum relaxation time, inertia can be neglected so that the velocities $\boldsymbol{v}_{i}$ of the beads are proportional to the forces acting on them [11]. Hence the beads follow the equations of motion

$$
\boldsymbol{v}_{i}=\sum_{j} \boldsymbol{\mu}_{i j} \boldsymbol{F}_{j} \text { with } \boldsymbol{F}_{j}=\boldsymbol{F}_{j}^{S}+\boldsymbol{F}_{j}^{B}+\boldsymbol{F}_{j}^{D},
$$

where the forces $\boldsymbol{F}_{j}^{S}, \boldsymbol{F}_{j}^{B}$, and $\boldsymbol{F}_{j}^{D}$ are given in the last section. They all depend on the beadslocations $\boldsymbol{r}_{i}$ and the dipolar forces also possess an explicite time dependence. The important quantities in our treatment are the mobilities $\boldsymbol{\mu}_{i j}$. Roper et al. considered the filament as a continuous line whose friction with the surrounding fluid is governed locally by two anisotropic friction coefficients for respective motions parallel and perpendicular to the local direction of the filament [24]. In our treatment, the anisotropic friction results from hydrodynamic interactions between neighboring beads. Moreover, hydrodynamic interactions between more distant beads are also taken into account. Since induced flow fields are long ranged (they decay as $1 / r$, where $r$ is the distance from a moving bead), this effect cannot be neglected. In general, hydrodynamic interactions constitute a complicated many-body problem [11], however, their leading contribution is given by twoparticle interactions. Moreover, if the particles are not too close to each other so that lubrication becomes important, the Rotne-Prager approximation can be employed [11, 31]. We use it in a version for spheres with different radii $a_{i}$ and $a_{j}$ [34]. Whereas all spheres in the filament have the same radius $a_{i}=a(1 \leq i \leq N)$, we also attach a larger sphere with radius $a_{0}$ to the filament to mimic its load. In the Rotne-Prager approximation, the self mobility is simply

$$
\boldsymbol{\mu}_{i i}=\mu_{0} \mathbf{1} \text { with } \mu_{0}=1 /\left(6 \pi \eta a_{i}\right),
$$

and the cross mobilities read

$\boldsymbol{\mu}_{i j}=\frac{1}{6 \pi \eta r_{i j}}\left[\frac{3}{4}\left(\mathbf{1}+\hat{\boldsymbol{r}}_{i j} \otimes \hat{\boldsymbol{r}}_{i j}\right)+\frac{1}{4} \frac{a_{i}^{2}+a_{j}^{2}}{r_{i j}^{2}}\left(\mathbf{1}-3 \hat{\boldsymbol{r}}_{i j} \otimes \hat{\boldsymbol{r}}_{i j}\right)\right]$.
To obtain the particle paths $\boldsymbol{r}_{i}(t)$ and thus the dynamics of the filament, we numerically integrate Eqs. (11) using the Euler method [35]. More accurate schemes such as the RungeKutta method that would allow larger time steps during the integration and thus speed up the simulations are not useful since the maximum time step for the integration is governed by the relaxation dynamics of local bending and stretching deformations. At the time step chosen to avoid numerical instabilities, the performance of the Euler method is comparable, e. g., to the fourth-order Runge-Kutta scheme. In concrete, the relative differences of the particle positions calculated with both methods are smaller than $10^{-4}$.

\section{Reduced Equations of Motion}

Our modelling of the filament comprises several parameters. To identify the essential parameters that govern the dynamics of the filament, we rescale the dynamic equations appropriately so that only reduced variables appear. For example, all lengths will be referred to the equilibrium length of the filament that we approximate by $L \approx N l_{0}$.

To arrive at reduced equations of motion, we first rescale the energies from section $\amalg \mathrm{A}$ with the help of characteristic quantities:

$$
H^{S}=\frac{1}{2} k \frac{L^{2}}{N} \tilde{H}^{S}, H^{B}=\frac{A}{L} \tilde{H}^{B}, H^{D}=\frac{4 \pi a^{6}}{9 \mu_{0}}(\chi B)^{2} \frac{N^{4}}{L^{3}} \tilde{H}^{D}
$$

where the reduced energies read:

$$
\begin{aligned}
\tilde{H}^{S} & =N \sum_{i}\left(\frac{l_{i}-l_{0}}{L}\right)^{2} \\
\tilde{H}^{B} & =N \sum_{i}\left(1-\hat{\boldsymbol{t}}_{i} \cdot \hat{\boldsymbol{t}}_{i+1}\right) \\
\tilde{H}^{D} & =\frac{1}{N^{4}} \sum_{i \neq j} \frac{1-3\left(\hat{\boldsymbol{r}}_{i j} \cdot \hat{\boldsymbol{p}}\right)^{2}}{\left(r_{i j} / L\right)^{3}} .
\end{aligned}
$$

The prefactor $k L^{2} / N$ of $\tilde{H}^{S}$ in Eqs. (14) is essentially the stretching modulus of an elastic rod and $\tilde{H}^{S}$ averages the square of the strain variable over the whole rod. The bending free energy of the filament with a uniform curvature $L / \sqrt{2}$ amounts to $A / L$ and the prefactor of $\tilde{H}^{D}$ gives the order of magnitude of the energy of $N$ interacting dipoles. To compare these characteristic energies to each other, we introduce the reduced magnetic field $B_{s}[36]$ and the parameter $k_{s}$ :

$$
\begin{aligned}
& B_{s}=\left(\frac{\frac{4 \pi a^{6}}{9 \mu_{0}}(\chi B)^{2} \frac{N^{4}}{L^{3}}}{A / L}\right)^{1 / 2}=\frac{2 \pi^{1 / 2} a^{3} \chi N}{3 \mu_{0}^{1 / 2} l_{0} A^{1 / 2}} B \\
& k_{s}=\frac{k L^{2} / N}{A / L}=\frac{N^{2} l_{0}^{3}}{A} k .
\end{aligned}
$$

In the following, we will characterize the strength of the magnetic field by $B_{s}$, the parameter $k_{s}$ will basically be kept constant.

Whereas the scaling factors in Eqs. (14) determine the magnitude of the energies, the reduced energies of Eqs. (15)-117 
distinguish between different types of stretching and bending deformations and arrangements of dipoles along the filament. For sufficiently large $L \approx N l_{0}$, they tend to constant values $\tilde{H}_{\infty}^{S}, \tilde{H}_{\infty}^{B}$, and $\tilde{H}_{\infty}^{D}$. We checked how finite size effects from the ends of the filaments influence these constant values. Bending the filament to a semicircle, the deviation from $\tilde{H}_{\infty}^{B}$ is around $1 \%$ when $N=20$, a typical number of beads in the superparamagnetic filament both in experiments and in our simulations. On the other hand, for the same number of beads $\tilde{H}^{D}$ for dipoles aligned along a straight line deviates from $\tilde{H}_{\infty}^{D}$ by around $10 \%$ due to the long range nature of the dipolar interaction.

To formulate the reduced equations of motion, we rescale length, time, and velocity according to

$$
\tilde{\boldsymbol{r}}=\boldsymbol{r} / L, \tilde{t}=\omega t, \frac{d \tilde{\boldsymbol{r}}}{d \tilde{t}}=\frac{1}{\omega L} \frac{d \boldsymbol{r}}{d t},
$$

where $\omega$ is the frequency of the actuating magnetic field. The reduced forces and mobilities follow from

$$
\widetilde{\boldsymbol{F}}_{i}=\frac{\boldsymbol{F}_{i}}{A / L^{2}}, \quad \tilde{\boldsymbol{\mu}}_{i j}=6 \pi \eta a \frac{L}{l_{0}} \boldsymbol{\mu}_{i j} .
$$

Note that $6 \pi \eta a L / l_{0}$ is the total friction coefficient of the filament without taking into account hydrodynamic interactions. Applying the rescaled quantities to Eqs. 11 and using Eqs. (14), (18), and (19), one arrives at the reduced equation of motion for bead $i$ :

$$
\frac{d \tilde{\boldsymbol{r}}_{i}}{d \tilde{t}}=S_{p}^{-4} \sum_{j} \tilde{\boldsymbol{\mu}}_{i j} \widetilde{\boldsymbol{F}}_{j}
$$

with

$$
\widetilde{\boldsymbol{F}}_{j}=-\nabla_{\tilde{\boldsymbol{r}}_{j}}\left(\tilde{H}^{B}+k_{S} \tilde{H}^{S} / 2+B_{S}^{2} \tilde{H}^{D}\right) .
$$

The important parameter $S_{p}$ in Eqs. (22), introduced first by Wiggins and Goldstein [23, 37] and termed sperm number by Lowe [26], is defined via

$$
S_{p}=\left(\frac{6 \pi \eta \frac{a}{l_{0}} \omega L^{4}}{A}\right)^{1 / 4}=\frac{L}{l_{h}} .
$$

It compares the frictional to the bending forces and completely determines the dynamics of an elastic filament in a viscous environment in the so-called resistive-force theory of slender bodies [38], where the friction with the surounding fluid is described by two local friction coefficients per length, $\gamma_{\|}$and $\gamma_{\perp}$, for respective motions parallel and perpendicular to the local axis of the filament. Our definition of $S_{p}$ agrees with the one of Wiggins and Goldstein [23, 37] when we make the reasonable identification $\gamma_{\perp}=6 \pi \eta a / l_{0}$. An immediate interpretation of $S_{p}=L / l_{h}$ is given with the help of the elastohydrodynamic penetration length $l_{h}$ [23, 37]. Consider a sufficiently long filament $\left(L \gg l_{h}\right)$ whose one end undergoes an oscillation with frequency $\omega$. Then $l_{h}$ is the length on which the oscillation penetrates into the filament. On the other hand, if $L \ll l_{h}$, the filament oscillates as a whole like a rigid rod.

Other important quantities are the strength of the actuating magnetic field quantified by the parameter $B_{s}$ and its time protocol, which we will concretize in section $\llbracket$ The reduced stretching constant $k_{s}$ is always chosen such that variations in the length of the filament are smaller than $10 \%$.

We already mentioned that the dynamics of a continuous elastic filament described within the resistive-force theory is completely determined by the sperm number $S_{p}$, which especially incorporates the influence from the filament length. In the superparamagnetic filament simulated by us through a discretized model, finite-size effects are present, as already discussed for the bending and dipolar energies. Hydrodynamic interactions are of longer range than dipolar interactions and we therefore discuss shortly how variations in the length of the filament or in the number of beads influence the dynamics for constant $S_{p}, B_{s}$, and $k_{s}$. We keep the strength of the magnetic field constant but let its direction oscillate in the $y z$ plane around the $z$ axis with an angular amplitude of $50^{\circ}$. We solve the equations of motion (11) for real parameters and change the length of the filament for constant equilibrium-bead distant $l_{0}$ by choosing the particle numbers $N=20,40$, and 80 . The reduced parameters are kept fixed at the values $S_{p}=7.7$, $B_{s}=6.6$, and $k_{s}=4500$ by changing, respectively, the frequency $\omega$ of the oscillating magnetic field, its strength $B$, and the real spring constant $k$. Figure 2a) shows snapshots of the different filaments in the $y z$ plane at the same moments within the period of the oscillating field. Hydrodynamic interactions are completely switched off. For $N=40$ and 80 the configuration of the filaments are basically identical whereas for $N=20$ one realizes small differences due to the finite-size effects from the dipolar interactions as discussed above. In Fig. $2 \mathrm{~b}$ ) the configurations are shown at the same moments within the period as in Fig. 22 but now with hydrodynamic interactions switched on. They clearly have a pronounced effect on the oscillating shape of the filament. Since the beads move collectively through the fluid, hydrodynamic interactions reduce, roughly speaking the local friction with the surrounding fluid. The filament can more easily follow the oscillating magnetic field direction and, as a result, it is less bent. The finite-since effect is more pronounced compared to Fig. 2a) but does not change the configurations significantly. So when in the following all our simulations are done with $N=20$ to reduce computing time, we are sure that the dynamics of the filament is mainly described by the sperm number $S_{p}$, the reduced strength $B_{s}$ of the magnetic field, and its time protocol.

\section{STUDY OF THE ARTIFICIAL SWIMMER}

The actuated superparamagnetic filament in Fig. 2 does not move on average in one direction since it performs a reciprocal motion. This symmetry is broken when a load is attached to one end of the filament as demonstrated by the wonderful experiments of Dreyfus et al. where the load is a red-blood cell [2]. In the following we study such an artificial swimmer in detail. As illustrated in Fig. 3, we actuate the filament with a magnetic field $\boldsymbol{B}(t)$ whose strength is constant but whose direction oscillates about the $z$ axis with an angle $\varphi(t)=\varphi_{\max } \sin (\omega t)$ (this time protocol differs from the one used in Ref. [2]). As a result, the swimmer will move with 

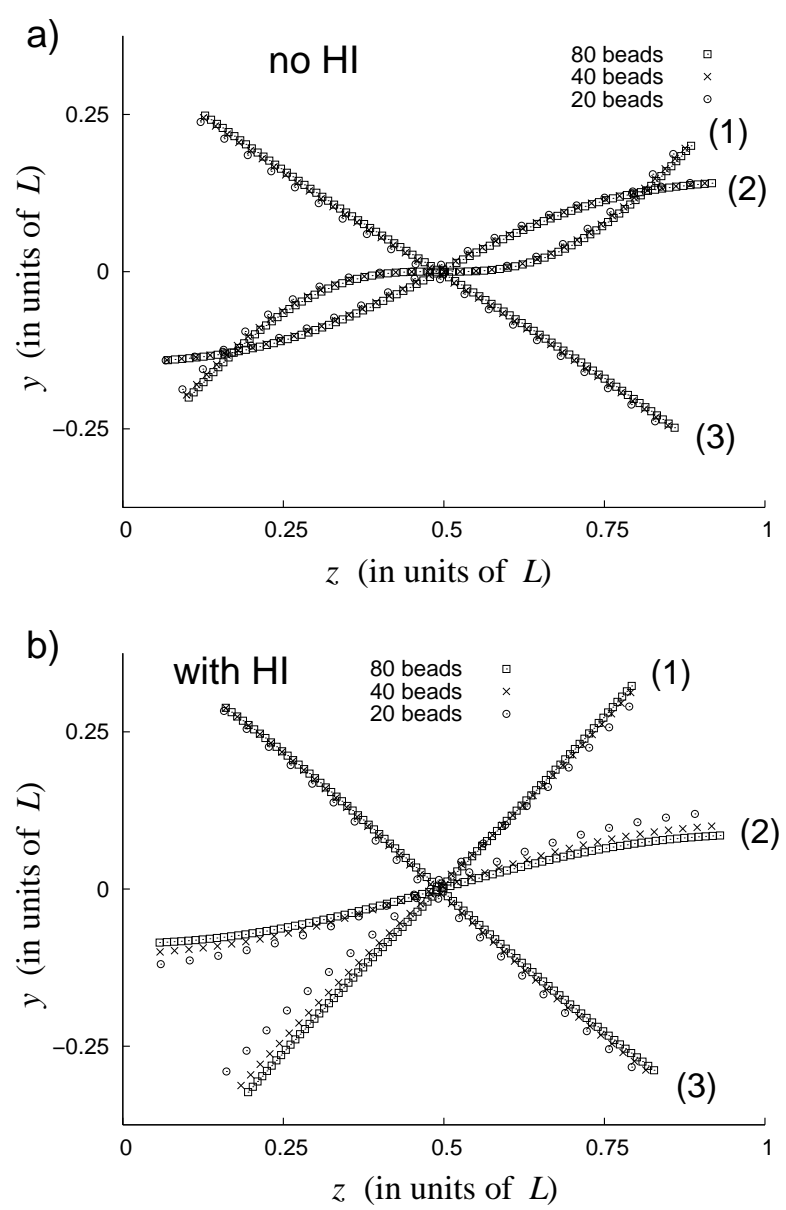

FIG. 2: Configurations of the superparamagnetic filament at the same moments within one period of the oscillating magnetic field. The reduced parameters $S_{p}, B_{s}$, and $k_{s}$ are kept constant while the number of beads is changed. a) without hydrodynamic interactions, b) with hydrodynamic interactions. The numbers at the snapshots indicate the pair of variables $\varphi, \omega t /(2 \pi)$ for the time protocol of the oscillating magnetic field, as illustrated in Fig. 3 (1) $50^{\circ}, 0.25$; (2) $0^{\circ}, 0.5$; (3) $-35^{\circ}, 0.88$.

an average velocity $\overline{\boldsymbol{v}}$ along the $z$ axis. However, in contrast to spermatozoa, where the head is pushed forward by damped waves travelling from the head to the tail [14, 15], the superparamagnetic filament drags the passive load behind itself by performing a sort of paddle motion with its free end as indicated in Fig. 3 Note that the direction can be reversed for certain parameters when the load particle also becomes superparamagnetic, but we will not study this possibility further. In our approach, hydrodynamic interactions between the constituent beads are essential for the artificial swimmer to move forward. In particular, they produce local friction coefficients $\gamma_{\|}$and $\gamma_{\perp}$ for respective motions parallel and perpendicular to the filament's local axis that are not equal, $\gamma_{\|} \neq \gamma_{\perp}$. This is a necessary prerequisit for swimming [27]. To implement hydrodynamic interactions, we use the Rotne-Prager approximation for the mobilities as introduced in Eqs. (12) and (13) in the form that also allows for hydrodynamic interactions between the filament's beads and the larger load particle. We

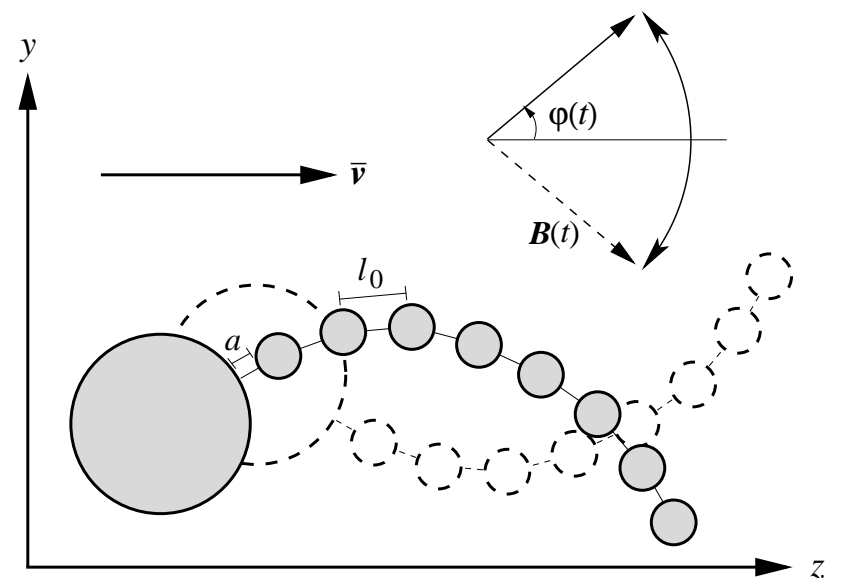

FIG. 3: For the artificial swimmer, a larger load particle is attached to the filament. It is actuated by a magnetic field whose direction is oscillating about the $z$ axis with $\varphi(t)=\varphi_{\max } \sin (\omega t)$. The two configurations of the swimmer are schematic drawings for positive and negative $\varphi(t)$.

simulate the artficial swimmer by solving the equations of motion (11) numerically for two sets of parameters summarized in Appendix A The first set is close to the realization of the artificial swimmer in Ref. [2]. Since we already now that the filament itself is governed by the dimensionless parameters $S_{p}, B_{s}$, and $k_{s}$, we present our results as functions of the sperm number $S_{p}$ and the reduced field strength $B_{s}$. In addition, we also show that the angular amplitude $\varphi_{\max }$ of the magnetic-field oscillations and the size $a_{0}$ of the load particle influence the dynamics of the swimmer.

We discuss the performance of the swimmer by studying in detail its average speed $\bar{v}$ and its efficiency to move a load. The speed $\bar{v}$ is calculated by averaging the velocity $\boldsymbol{v}_{0}$ of the load particle over one actuation cycle:

$$
\overline{\boldsymbol{v}}=\frac{1}{T} \int_{\tau}^{\tau+T} \boldsymbol{v}_{0}(t) d t
$$

where $T=2 \pi / \omega$. In order to obtain a reliable $\overline{\boldsymbol{v}}$ from Eq. 25], it is important that the swimmer has reached a steady state at time $\tau$ so that the swimming does not depend any more on initial conditions. For small frequencies $\left(S_{p} \approx 1\right)$, this state is reached rather quickly after one actuation cycle but needs several cycles when $S_{p}$ is increased. Secondly, we define the efficiency of the swimmer to transport a load by comparing the energy dissipated by the load, when moved uniformly with velocity $\overline{\boldsymbol{v}}$, to the total energy dissipated by the swimmer:

$$
\xi=\frac{6 \pi \eta a_{0} \bar{v}^{2}}{\overline{\sum_{i=0}^{N} \boldsymbol{F}_{i} \cdot \boldsymbol{v}_{i}}},
$$

where the bar in the denominator means average over one actuation cycle. The efficiency $\xi$ indicates how much energy from the total energy used to actuate the swimmer is employed to move the load particle forward with velocity $\overline{\boldsymbol{v}}$.

In Fig. 4, we plot the mean velocity $\bar{v}=|\overline{\boldsymbol{v}}|$ and the efficiency $\xi$ as a function of the sperm number $S_{p}$. For the simulations, the realistic parameter set I was used. The curve for 
$\bar{v} /(L \omega)$ in the upper graph of Fig. 4 follows the same behavior as observed in Ref. [26, 27], where the elastic filament is driven by an oscillating torque acting on one of its ends. For small sperm numbers around $S_{p}=3$, the reduced velocity is small since the superparamagnetic filament behaves nearly like a rigid rod, as illustrated by the inset on the lower left. It shows snapshots of the filament for $S_{p}=3$. As already mentioned, the oscillating motion of a rigid rod is reciprocal and therefore cannot produce a directed motion of the swimmer. Increasing the sperm number, e. g., by increasing the frequency $\omega$, speeds up the artificial swimmer. At the maximum value of $\bar{v} /(L \omega)$ at around $S_{p}=6$, the increased friction with the surrounding fluid is able to bend the whole filament (see upper inset) which obviously promotes a high swimming velocity. Further increase in $S_{p}$ leads to a decrease in $\bar{v} /(L \omega)$; due to the strong friction with the fluid the whole filament cannot follow the magnetic field and only a small wiggling of its free end remains. The efficiency as a function of $S_{p}$ shows a similar behavior as $\bar{v} /(L \omega)$ that is not completely surprising: oscillating a rigid rod (small $S_{p}$ ) or fast wiggling of the filament (large $S_{p}$ ) dissipates energy but does not produce an effective motion. So one expects a maximum of $\xi$ close to the maximum of $\bar{v} /(L \omega)$ since $\xi$ is determined by $\bar{v}^{2}$. Note that the small absolute efficiency $\left(\xi_{\max }=1.58 \cdot 10^{-3}\right)$ is due to the fact that a large amount of energy is dissipated by the motion of the filament. The shape of the velocity curve changes when absolut velocities are plotted, as demonstrated in the lower graph of Fig. (4) At $S_{p}=3$, the absolute velocity is nearly zero (due to the small frequency) and the maximum is shifted to a larger value around $S_{p}=7.5$. Interestingly, the absolute velocities of the oscillating filaments at $S_{p}=6$ and 12 do not differ so much, as first implied by the upper graph. This suggests that the absolute swimming velocity depends on two factors: (1) the shape of the oscillating filament, where bending the whole filament favors large velocities, and (2) the oscillation frequency. The latter point leads to the very slow decrease of $\bar{v}$ as a function of $S_{p}$ in the lower graph. A comparison with the narrow maximum of $\xi$, however, shows that a lot of energy is dissipated at large $S_{p}$ in the small wiggling motion of the filament. So operating the artificial swimmer at around $S_{p}=7$ between the two close maxima ensures highest swimming velocities with very efficient energy consumption.

In Fig. 5 we present reduced velocities and efficiencies as a function of $S_{p}$ for several reduced field strengths $B_{s}$, which we simulated with parameter set II. We observe that with increasing $B_{s}$ the maxima of both the velocity and the efficiency curves move to larger sperm numbers. We understand this since larger magnetic fields mean stronger alignment of the dipoles and, therefore, larger resistance to bending. Both, maximium velocity and efficiency, only exhibit a small dependence on $B_{s}$. After a slight increase of the maximum velocity, it slightly decreases to a constant value (not shown in the graph). Note that the reduced velocities are larger by a factor of 1.2 compared to Fig. (4) that we attribute to the larger angular amplitude $\varphi_{\max }=57^{\circ}$ compared to $\varphi_{\max }=45^{\circ}$ used in Fig. 4. Finally, Fig. 6]summarizes the dependence of the absolut swimming velocity on sperm number and reduced field calculated with parameter set II. For increasing field strength at constant $S_{p}$, the graph shows a strong increase of the swim-
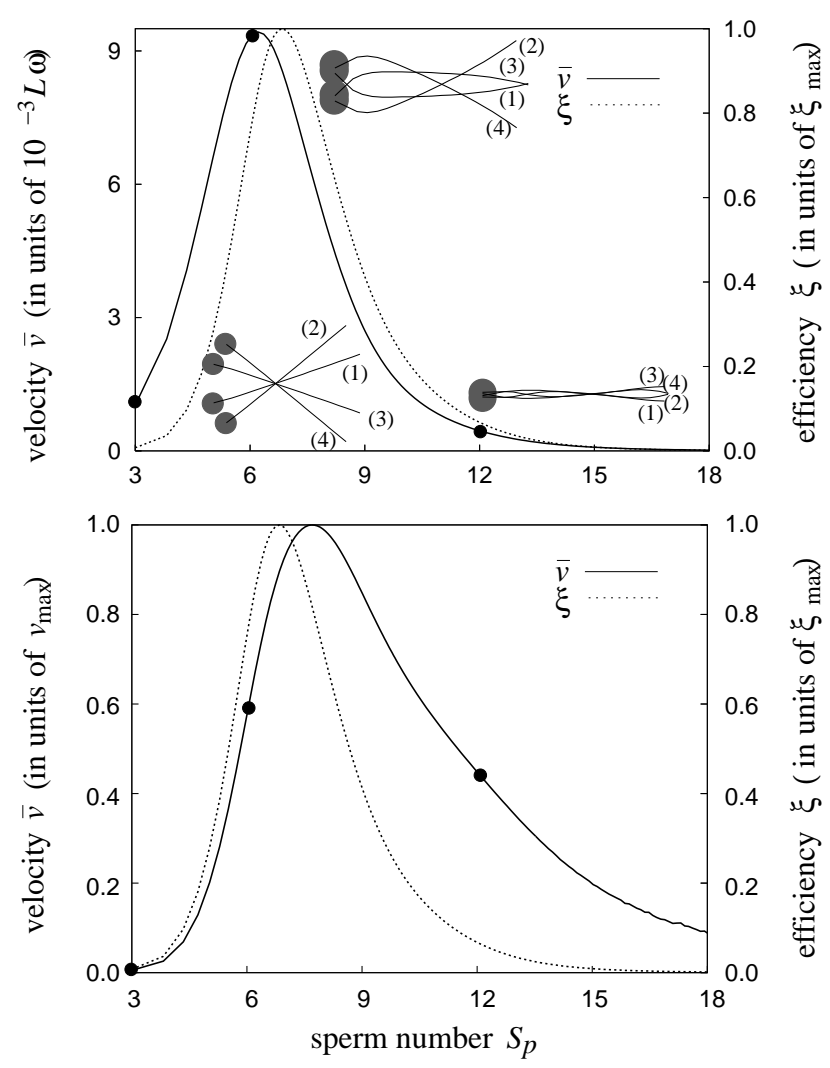

FIG. 4: Swimming velocity $\bar{v}$ and efficiency $\xi$ in units of $\xi_{\max }=$ $1.58 \cdot 10^{-3}$ as a function of sperm number $S_{p}$ for reduced magneticfield strength $B_{S}=5.76$. Upper graph: reduced velocity $\bar{v} /(L \omega)$, lower graph: absolut velocity $\bar{v}$ in units of $v_{\max }=5.56 \cdot 10^{-5} \mathrm{~m} / \mathrm{s}$. The insets show several snapshots of the filament's configuration for $S_{p}=3,6$ and 12, respectively, indicated by the dots. The numbers at the snapshots indicate the pair of variables $\varphi, \omega t /(2 \pi)$ for the time protocol of the oscillating magnetic field, as illustrated in Fig. 3. (1) $20^{\circ}, 0.07$; (2) $40^{\circ}, 0.17$; (3) $-20^{\circ}, 0.57$; (4) $-40^{\circ}, 0.67$. Parameter set I was used in simulations.

ming velocity. At small $S_{p}$ a subsequent saturation at a constant value is visible. Note that the maximum velocity for constant $B_{s}$ is shifted to higher sperm numbers when $B_{s}$ increases. This explains why the the maximum reduced velocity $\boldsymbol{v} /(L \omega)$ in Fig. [5]does not exhibit a strong dependence on $B_{s}$.

The swimming velocity $\bar{v}$ clearly depends on the size or the radius $a_{0}$ of the load particle. In the upper graph of Fig. 7 , we plot its absolute value as a function of sperm number $S_{p}$ and $a_{0}$ for a constant field strength $B_{s}=5.76$ calculated with parameter set I. There is a pronounced maximum at $S_{p}=8$ and $a_{0} \approx 3 a$ (indicated by a filled circle), where $a$ is the radius of the superparamagnetic beads in the filament. The velocity $\bar{v}$ decreases for large $a_{0}$ since the load becomes too heavy to be efficiently moved by the oscillating filament. On the other hand, when $a_{0}$ approaches $a$, we also expect small swimming velocities since the asymmetry of the swimmer becomes small. However, even at $a_{0}=a$ the swimming velocity is not zero and therefore the swimmer still performs a non-reciprocal motion. The reason is that the load particle is not superparamagnetic and, therefore, a small asymmetry 


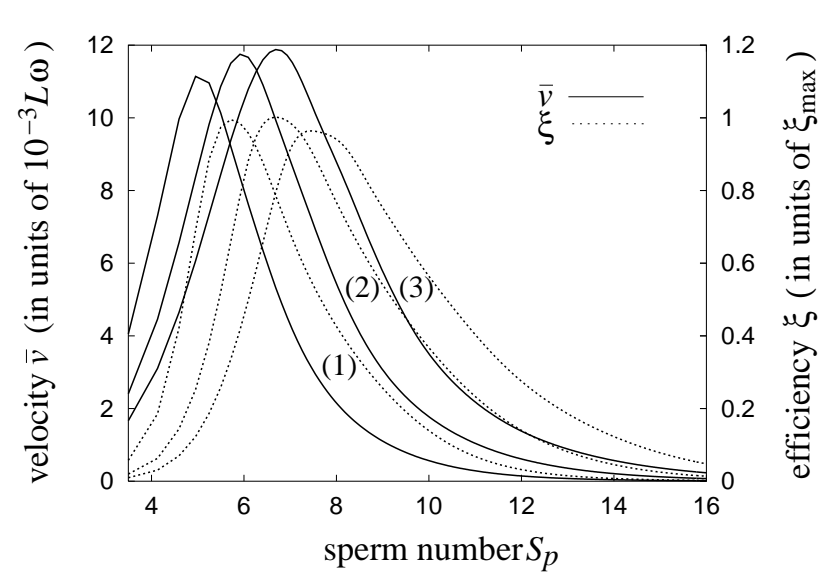

FIG. 5: Reduced swimming velocity $\bar{v} /(L \omega)$ and efficiency $\xi$ in units of $\xi_{\max }=1.87 \cdot 10^{-3}$ as a function of sperm number $S_{p}$ for several reduced magnetic-field strengths: (1) $B_{s}=4.2$, (2) $B_{s}=6.07$, and (3) $B_{s}=7.93$. Parameter set II was used in simulations.

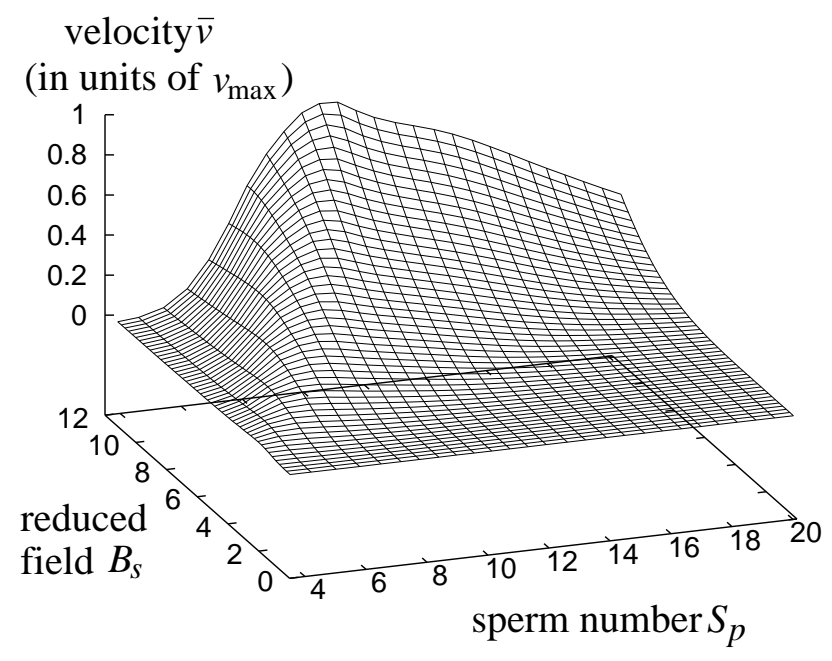

FIG. 6: Absolute swimming velocity $\bar{v}$ in units of the maximal value $v_{\max }$ as a function of sperm number $S_{p}$ and reduced magnetic-field strength $B_{s}$. Parameter set II was used in simulations.

remains. The plot for the reduced velocity $\bar{v} /(L \omega)$ looks similar to the lower graph of Fig. 7, the absolute maximum is not that pronounced and the maxium for constant $a_{0}$ moves to smaller sperm numbers when $a_{0}$ is increased. The lower graph shows how the efficiency $\xi$ behaves as a function of $S_{p}$ and $a_{0}$. The absolute maximum, indicated by an open circle, is at $S_{p}=6.6$ and $a_{0} \approx 5 a$. For comparison the location of the maximum of the swimming velocity is again shown by the filled circle. Since it is relatively sharp, one has to choose a compromise for the performance of the swimmer between the largest swimming velocity and the best efficiency. The location of the maximum velocity certainly depends on the magnetic-field strength. We expect that it is shifted to larger radii $a_{0}$ when $B_{s}$ increases. However, we have not studied this dependence in detail.

So far, the angular amplitude $\varphi_{\max }$ of the oscillating field was always chosen sufficiently small so that the swimmer was
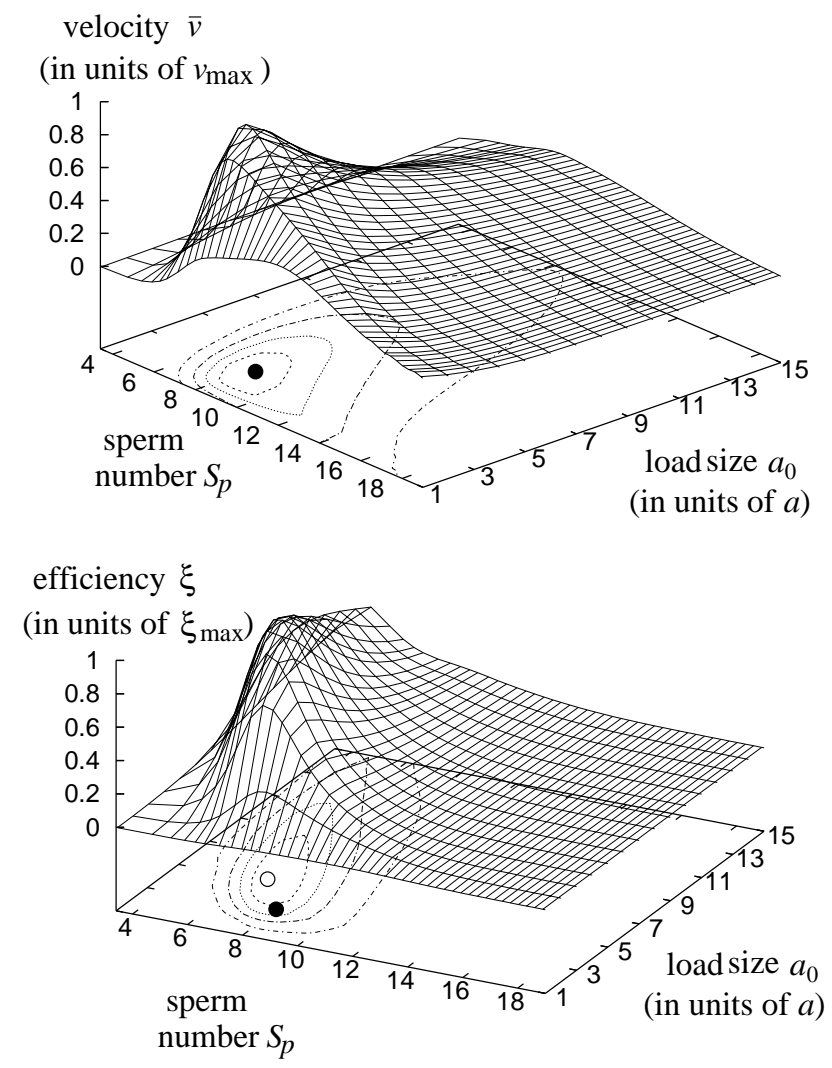

FIG. 7: Swimming velocity $\bar{v}$ (in units of $v_{\max }=7.31 \cdot 10^{-5} \mathrm{~m} / \mathrm{s}$ ) and efficiency $\xi$ (in units of $\xi_{\max }=1,54 \cdot 10^{-3}$ ) as a function of sperm number $S_{p}$ and load size $a_{0}$ in units of $a$. The filled and open circles indicate, respectively, the absolute maxima of $\bar{v}$ and $\xi$. $B_{s}=5.76$ and parameter set I was used in simulations.

moving along the $z$ axis. For large $\varphi_{\max }$, however, the mean velocity $\overline{\boldsymbol{v}}$ assumes a non-zero angle $\psi$ with the $z$ axis. The upper graph of Fig. 8 summarizes our results. For $S_{p}$ larger than 7 and angular amplitudes beyond $\varphi_{\max }=70^{\circ}$, the swimming angle $\psi$ jumps from $0^{\circ}$ to $90^{\circ}$ and the swimmer thus moves perpendicular to the $z$ axis. Such an abrupt transition was also observed in the experiments by Dreyfus et al. [39]. It is associated with a broken symmetry since the swimmer could also move into the opposite direction with $\psi=-90^{\circ}$ depending on the initial condition. In the beginning the filament and magnetic field point along the $z$ axis. When the magnetic-field direction turns towards the upper-half space $(y>0)$, the filament follows. For large oscillation frequencies $\omega$ (large $S_{p}$ ), it cannot, however, follow the field direction into the lowerhalf space $(y<0)$ and $\psi=90^{\circ}$ results, as pictured in the third example of Fig. 9 For decreasing sperm number, this sharp transition becomes smoother involving swimming directions inclined relativ to the $z$ axis. Finally for $S_{p}<3$, the swimmer always swims along the $z$ axis by following the oscillating field as a nearly rigid rod with only small bending. The simulations of the swimmer's movements for $\psi \neq 0$ are very time consuming since the swimmer needs some time to reach a steady state. Several snapshots of its configurations for different parameters are summarized in Fig. 9 their locations in the graphs of Fig. 8 are indicated by filled black circles. The 

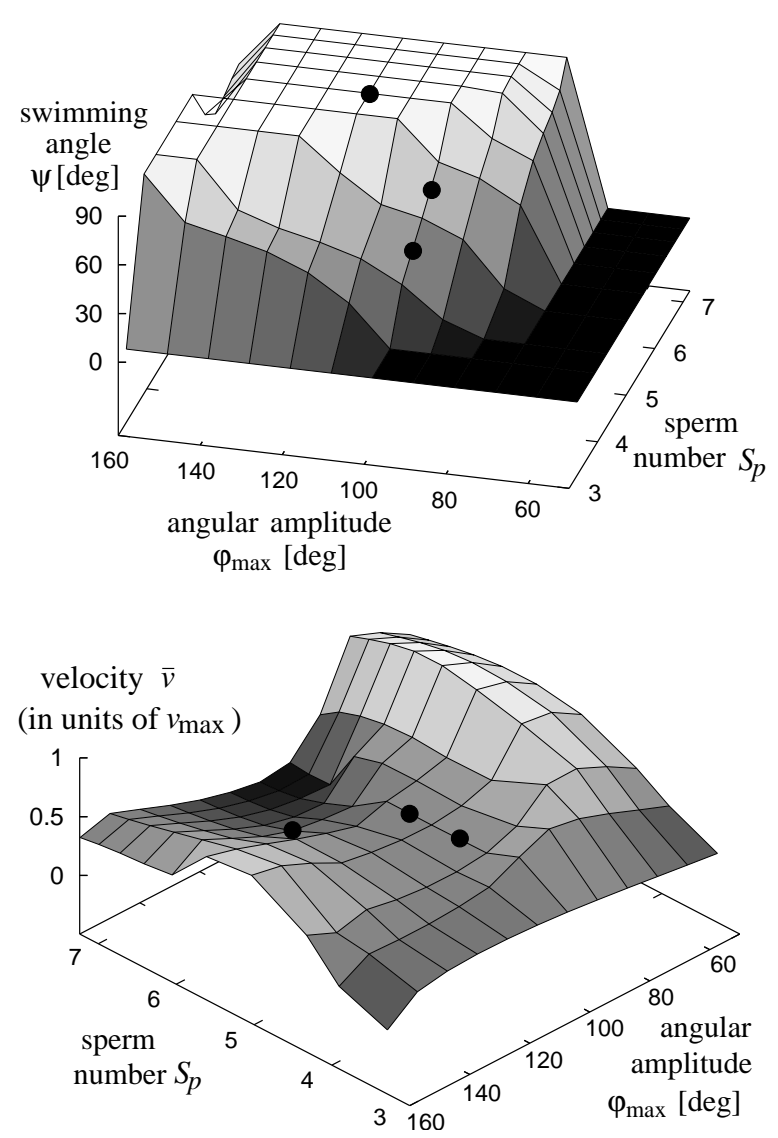

FIG. 8: Swimming velocity $\bar{v}$ (in units of $v_{\max }=5.57 \cdot 10^{-3} \mathrm{~m} / \mathrm{s}$ ) and the swimming angle $\psi$ (measured relative to the $z$ axis) as a function of sperm number $S_{p}$ and angular amplitude $\varphi_{\max }$ of the oscillating field. $B_{s}=3.81$ and parameter set II was used in simulations.

temporal evolution of the first and second configurations in Fig. 9 are the dynamic analog of the static hairpin structures that form when the aligning magnetic field is suddenly turned around by $90^{\circ}[19]$. Finally, the lower graph of Fig. 8 8 plots the swimmming velocity $\bar{v}$ as a function of $S_{p}$ and $\varphi_{\max }$. Note that the $S_{p}$ and $\varphi_{\max }$ axes are differently oriented compared to the upper graph. When the angular amplitude $\varphi_{\max }$ increases from $0^{\circ}$, we observe that $\bar{v}$ also increases (only partially shown in the graph). At $S_{p}=7$ and around $\varphi_{\max }=70^{\circ}$, a sharp decrease in $\bar{v}$ coincides with the abrupt change of the swimming direction from $\psi=0^{\circ}$ to $90^{\circ}$ in the upper graph. For decreasing $S_{p}$, the sharp drop in $\bar{v}$ becomes smaller due to the occurence of inclined swimming directions.

\section{CONCLUSIONS}

In this article, we have presented a detailed numerical study of a microscopic artificial swimmer realized recently by Dreyfus et al. in experiments [2]. The elastic superparamagnetic filament is modeled by a bead-spring configuration that also resists bending via a disrete version of the worm-like chain. Friction with the surrounding fluid is described by the single beads that also experience hydrodynamic interactions with

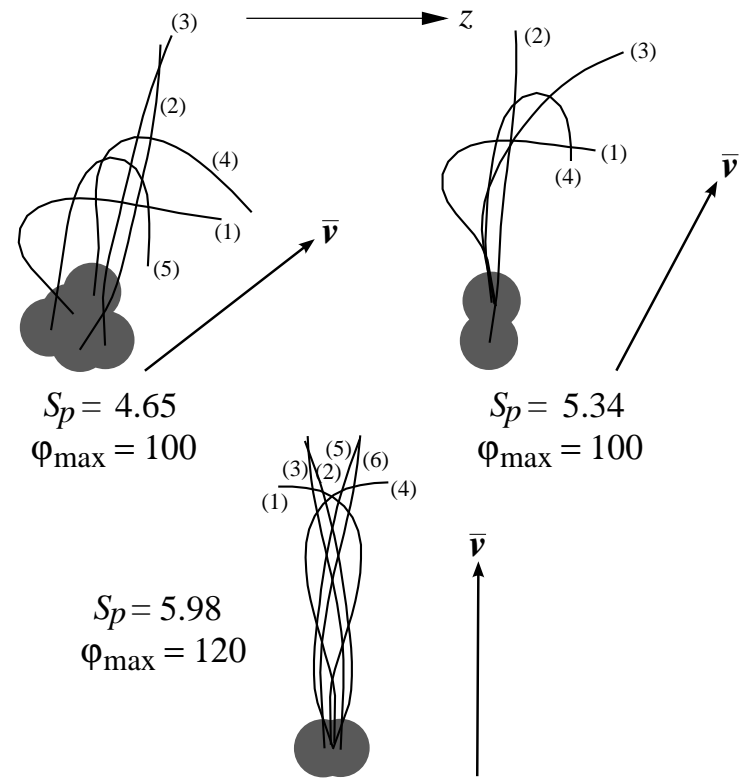

FIG. 9: Snapshots of the swimmer's configurations for different parameters giving rise to different swimming directions. Their locations in the graphs of Fig. 8 are indicated by filled black circles. The numbers at the snapshots indicate the pair of variables $\varphi, \omega t /(2 \pi)$ for the time protocol of the oscillating magnetic field. $S_{p}=4.65$ : (1) $0^{\circ}, 0.00$; (2) $95^{\circ}, 0.20$; (3) $58^{\circ}, 0.40$; (4) $-58^{\circ}, 0.60$; (5) $-95^{\circ}, 0.80$. $S_{p}=5.34$ : (1) $0^{\circ}, 0.00$; (2) $100^{\circ}, 0.25$; (3) $0^{\circ}, 0.50$; (4) $-100^{\circ}, 0.75$. $S_{p}=5.98$ : (1) $41^{\circ}, 0.06$; (2) $118^{\circ}, 0.22$; (3) $79^{\circ}, 0.39$; (4) $-37^{\circ}, 0.55$; (5) $-117^{\circ}, 0.72 ;(6)-82^{\circ}, 0.88$.

each other. The swimmer composed of the filament and an attached load is actuated by a magnetic field whose direction oscillates.

We show that the superparamagnetic filament, aside from finite-size effects, can be described by the dimensionless sperm number, the magnitude of the magnetic field, and the angular amplitude of the field's oscillating direction. We then study the mean velocity and the efficiency of the swimmer, which we define appropriately, as a function of these parameters and the size of the load particle. In particular, we clarify that the real velocity of the swimmer depends on two main factors namely the shape of the beating filament and the oscillation frequency. For given magnetic-field strength an optimum sperm number (or oscillation frequency) can be chosen such that mean velocity and efficiency are close to their maximum values. Whereas the maximum rescaled velocity $\bar{v} /(L \omega)$ as a function of the sperm number only exhibits a weak dependence on the magnetic-field strength $B_{s}$, the real maximum velocity $\bar{v}$ strongly increases with $B_{S}$ since its location is shifted to larger $S_{p} \propto \omega^{1 / 4}$. A study of the influence of the load size for a particular field strength reveals, the optimum load has to be chosen as a compromise between the largest swimming velocity and the best efficiency. For increasing angular amplitude of the field's oscillating direction, the direction of the swimming velocity changes in a symmetry-breaking transition that is sharp for large sperm numbers, becomes smoother for decreasing $S_{p}$, and ultimately vanishes around $S_{p}=3$. Accordingly, the jump in the swimming angle relative to the sym- 


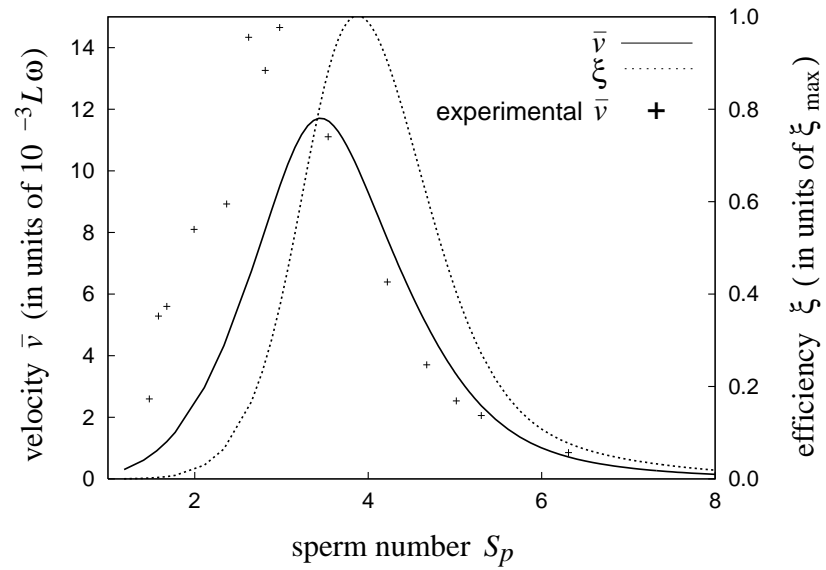

FIG. 10: Swimming velocity $\bar{v}$ and efficiency $\xi$ in units of $\xi_{\max }=$ $1.8 \cdot 10^{-3}$ as a function of sperm number $S_{p}$. Parameters are the same as the one used for the red experimental data points of Fig. 4 in Ref. [2]. The experimental points are included in this figure with the symbol + .

metry axis decreases from $90^{\circ}$ to $0^{\circ}$.

We also applied the time protocal of Ref. [2] (i.e., a constant $z$ component and an oscillating $y$ component of the magnetic field) to actuate the one-armed swimmer. For the parameters of the red experimental data points of Fig. 4 in Ref. [2], we find nearly quantitative agreement, as illustrated in Fig. 10 . To achieve this, we had to rescale the actuating magnetic field by a factor of 2.53 to account for the larger distance of the beads in our modelling and therefore to compensate for the weaker dipole interaction compared to the swimmer in Ref. [2]. This makes sense since the strength of the dipole interaction of neighboring dipoles is the important parameter for the actuation of the swimmer. Deviations between our simulations and the experimental results might be due to the fact that we use a spherical load particle compared to the oblate shape of the red blood cell used in Ref. [2] and that we neglect corrections to the actuating external field due to the induced dipole fields.

In Ref. [40], the authors envisage micro machines moving in blood vessels and doing necessary repair work. The onearmed swimmer offers an interesting possibility to propel such micro machines. Our numerical studies demonstrate that theoretical modelling helps to elucidate the basic features but also to optimize the performance of such micro machines.

\section{Acknowledgments}

We would like to thank J. Bibette and R. Dreyfus for making us familiar with the one-armed swimmer and together with M. Reichert for helpful discussions. H.S. acknowledges financial support from the Deutsche Forschungsgemeinschaft under Grant No. Sta 352/5-2. E.G. and H.S. thank the International Graduate College at the University of Konstanz for financial support.

TABLE I: Parameter sets I and II used in the numerical studies.

\begin{tabular}{ccc}
\hline parameter & set I & set II \\
\hline \hline$N$ & 20 & 20 \\
$a[\mu \mathrm{m}]$ & 0.5 & 2.25 \\
$a_{0}$ & $5 a$ & $8 a$ \\
$l_{0}$ & $3 a$ & $3 a$ \\
$\chi$ & 0.993 & 1.704 \\
$\eta\left[\mathrm{Ns} / \mathrm{m}^{2}\right]$ & $10^{-3}$ & $10^{-3}$ \\
$k[\mathrm{~N} / \mathrm{m}]$ & $1.5 \cdot 10^{-3}$ & 1.0 \\
$A[\mathrm{Nm}]$ & $4.5 \cdot 10^{-22}$ & $6.75 \cdot 10^{-18}$ \\
$\omega[2 \pi / s]$ & $1 \ldots 2285$ & $100 \ldots 9.4 \cdot 10^{4}$ \\
$\rightarrow S_{p}$ & $2.903 \ldots 20.074$ & $3.636 \ldots 20.134$ \\
$B[\mathrm{~T}]$ & 0.07 & $0.01 \ldots 0.49$ \\
$\rightarrow B_{s}$ & 5.76 & $0.233 \ldots 11.433$ \\
$\varphi_{\max }\left[{ }^{\circ}\right]$ & 45 & 57 \\
$\Delta t[\mathrm{~s}]$ & $10^{-6}$ & $10^{-8}$ \\
$n_{s}$ & 15 & 15 \\
\hline
\end{tabular}

\section{APPENDIX A: PARAMETER SETS}

In table I we summarize the parameters in set I and II used for our numerical studies. The oscillation frequency $\omega=2 \pi / T$ and the magnetic-field strength $B$ were varied to study the respective ranges of sperm number $S_{p}$ and reduced magnetic field $B_{s}$. The spring constant $k$, the time step $\Delta t$ for the Euler integration, and the number $n_{s}$ of simulation cycles were adjusted as necessary. Typical values are shown.
[1] D. Bray, Cell Movements: From Molecules to Motiliy, 2nd ed., Garland Publishing, New York (2001).

[2] R. Dreyfus, J. Baudry, M. L. Roper, M. Fermigier, H. A. Stone, and J. Bibette, Nature 437, 862 (2005).

[3] E. M. Purcell, Am. J. Phys. 45, 3 (1977).

[4] H. C. Berg, PNAS 93, 14225 (1996).

[5] A. Shapere and F. Wilczek, Phys. Rev. Lett. 58, 2051 (1987).

[6] H. A. Stone and A. D. T. Samuel, Phys. Rev. Lett. 77, 4102 (1996).

[7] L. E. Becker, S. A. Koehler, and H. A. Stone, J. Fluid Mech. 490, 15 (2003).
[8] J. E. Avron, O. Gat, and O. Kenneth, Phys. Rev. Lett. 93, 186001 (2004).

[9] A. Najafi and R. Golestanian, Phys. Rev. E 69, 062901 (2004).

[10] R. Dreyfus, J. Baudry, and H. A. Stone, Eur. Phys. J. B 47, 161 (2005).

[11] J.K.G. Dhont, An Introduction to Dynamics of Colloids (Elsevier, Amsterdam, 1996).

[12] H. C. Berg and R. A. Anderson, Nature 245, 382 (1973).

[13] H. C. Berg, E. coli in Motion, Springer Verlag, New York (2004).

[14] G. Taylor, Proc. R. Soc. A 209, 447 (1951). 
[15] J. Gray and G. J. Hancock, J. Exp. Biol. 32, 802 (1955); P. Satir, J. Cell Biol. 39, 77 (1968).

[16] R. W. Linck, Cilia and Flagella in Encyclopedia of Life Sciences, Nature Publishing Group (2001), www.els.net.

[17] S. Camalet, F. Jülicher, and J. Prost, Phys. Rev. Lett. 82, 1590 (1999).

[18] S. Camalet and F. Jülicher, New J. Phys. 2, 24 (2000).

[19] C. Goubault, P. Jop, M. Fermigier, J. Baudry, E. Bertrand, and J. Bibette, Phys. Rev. Lett. 91, 260802 (2003).

[20] L. Cohen-Tannoudji, E. Bertrand, L. Bressy, C. Goubault, J. Baudry, J. Klein, J.-F. Joanny, and J. Bibette, Phys. Rev. Lett. 94, 038301 (2005).

[21] A. Koenig, P. Hébraud, C. Gosse, R. Dreyfus, J. Baudry, E. Bertrand, and J. Bibette, Phys. Rev. Lett. 95, 128301 (2005).

[22] S. L. Biswal and A. P. Gast, Phys. Rev. E 68, 021402 (2003); Phys. Rev. E 69, 041406 (2004).

[23] C. H. Wiggins and R. E. Goldstein, Phys. Rev. Lett. 80, 3879 (1998).

[24] M. L. Roper, R. Dreyfus, J. Baudry, M. Fermigier, J. Bibette, and H. A. Stone, to be published in J. Fluid Mech. (2006).

[25] A. Cebers, J. Phys.: Condens. Matter 15, S1335 (2003); Curr. Opin. Coll. Interface Science 10, 167 (2005); Magnetohydrodynamics 41, 63 (2005).

[26] C. P. Lowe, Phil. Trans. R. Soc. Lond. B 358, 1543 (2003).

[27] M. C. Lagomarsino, F. Cap uani, C. P. Lowe, J. Theor. Biol. 222, 215 (2003).

[28] O. Kratky and G. Porod, Recl. Trav. Chim. Pays-Bas 68, 1106 (1949).
[29] Since the filament is actuated by an external field that only oscillates in a plane, twisting of the filament does not occur and, therefore, does not have to be taken into account when formulating the free energy of the system.

[30] L. D. Landau and E. M. Lifschitz, Lehrbuch der Theoretischen Physik Band VII: Elastizitätstheorie (Akademie Verlag, Berlin, 1991)

[31] In our modelling, we use a center-to-center distance $3 a$ of the particles in the filament (where $a$ is the particle radius). In this case, the Rotne-Prager approximation deviates from the full multipole results of Ref. [32] by at most 5\%. We checked this by using the program Hydrolib (see Ref. [33]).

[32] B. Cichocki, B. U. Felderhof, K. Hinsen, E. Wajnryb, and J. Blawdziewicz, J. Chem. Phys. 100, 3780 (1994).

[33] K. Hinsen, Comput. Phys. Commun. 88, 327 (1995).

[34] D. J. Jeffrey and Y. Onishi, J. Fluid Mech. 139, 261 (1983).

[35] W. H. Press, S. A. Teukolsky, W. T. Vetterling, and B. P. Flannery, Numerical Recipes in C: The Art of Scientific Computing, Cambridge University Press, Cambridge (1992).

[36] $B_{s}^{2}$ is proportional to the magnetoelastic number used in Refs. [2, 24].

[37] C. H. Wiggins, D. Riveline, A. Ott, and R. E. Goldstein, Biophys. J. 74, 1043 (1998).

[38] C. Brennen and H. Winet, Ann. Rev. Fluid Mech. 9, 339 (1977).

[39] R. Dreyfus, private communication (2005).

[40] K. Ishiyama, M. Sendoh, A. Yamazaki, M. Inoue, K. I. Arai, IEEE Trans. Mag. 37, 2868 (2001). 